\title{
Toward the Future: Multipurpose 3D Printed Prosthetic Arm
}

\author{
Kiran S Swami ${ }^{1}$, S B Dandagi ${ }^{2}$, V M Jamandar ${ }^{3}$, K B Mali ${ }^{4}$ \\ PG Student, Product Design and Manufacturing Department, Vishvesvaraya Technological University, Belgaum, India ${ }^{1}$ \\ Senior Professor\& PG Coordinator, Product Design and Manufacturing Department, Vishvesvaraya Technological \\ University, Belgaum, India ${ }^{2}$ \\ Assistant Professor, Mechanical department, Dr. Daulatrao Aher Colloge of Engineering, Karad, India ${ }^{3}$ \\ Industrialist, Spark Industries, Karad, India ${ }^{4}$
}

\begin{abstract}
Prosthetics is that area of medical industry which deals with artificial limbs. These are used for those who lost their limbs. But there are many people who have their limbs and cannot use them due to neurological disorders. The current work aims at achieving a solution to the people who have neurological Disorder problem by using Additive manufacturing technology i.e. 3D Printing. The mechanism is initially modeled in Unigraphics. The models are then fabricated in 3D printing using PLA/ABS material. Then the fabrication is again redesigned to be mounted upon a robot for imaginative multipurpose use.
\end{abstract}

Keywords: Prosthetics, 3D Printing, PLA, Additive Manufacturing, Fused Deposition Method (FDM), Robots.

\section{INTRODUCTION}

Prosthetics is area of large importance in Medical industry. customized prosthetics, implants, and anatomical models; There are many physically challenged persons who require 3. Pharmaceutical research regarding drug dosage forms, artificial limbs and a lot of work is performed in this delivery, and discovery. region.

All the work detailed in his thesis shows that the existing prosthetic arms are for those who lost their limbs. But there are many people who have their limbs and yet cannot use them due to neurological disorders. The aim of the current work is to make use of now emerging Additive manufacturing technique i.e. $3 \mathrm{D}$ printing for generating a solution to the foresaid problem.

According to this paper, Project developer typically will use 3D printing to identify design flaws, make changes and then make second-generation machined parts or invest in tooling to create injection-molded parts. Use of rapid prototyping technique i.e Fused deposition modelling the parts will be created and tested basically for fitment.

Then the part is been mounted on a robot with which one can achieve a large number of application( Household and also Industrial).

Dr.ShyamThangaraju, et al [1] discussed the application of $3 \mathrm{D}$ printing the area of medicine. These include precise arrangement of human embryonic stem cells, printing organs, blood vessels, skin etc, and orthopedic applications. C. Lee Ventola [2] detailed the use of 3D printing in medical applications. According to this author, Medical Applications is classified into several types namely: 1 . tissue and organ fabrication; 2 . creation of
The benefits of 3D printing in medical industry are also listed. They include: the customization and personalization of medical products, drugs, and equipment, costeffectiveness; increased productivity, democratization of design and manufacturing and enhanced collaboration.

\section{OBJECTIVE OF THE WORK}

As mentioned earlier, the current work is aimed to generate a supporting mechanism for physically disabled persons who don't have an part of hand above the wrist. In other words, the work is related to prosthetic arm. Very little research is carried out in this area.

The current work is aimed at designing and fabricating a supporting mechanism for those who have either lost or not grown as arm due to neurological disorders. Unigraphicsis used for modeling the support mechanism and Additive manufacturing (Fused deposition modelling) is used for fabrication. PLA/ABS is used for 3D printing.

\section{3D PRINTING}

3D printing also known as Additive Manufacturing is a process of manufacturing $3 \mathrm{D}$ objects. $3 \mathrm{D}$ printing is basically a Stereolithographic process which is invented by Chuck Hull [17]. Stereolithography is the first commercial Rapid prototyping process. A range of $3 \mathrm{D}$ 
IARJSET

printing technologies now exist which are used depending The process of preparing the model for printing involves on the application. Fused Deposition Modeling (FDM)[18] three steps. Exporting the 3D model into '.stl' file, using is now commonly used 3D printing technique. FDM works the generated 'stl' file for slicing the model and thus on an "additive" principle by laying down material in generating the toolpath, exporting the toolpath into Glayers; a plastic filament or metal wire is unwound from a Code. Most of the FDM 3D printers use conventional coil and supplies material to produce a part. The filament Gcodes. Various software tools are available for slicing is passed through a heated nozzle. During this passage, the the $3 \mathrm{D}$ model.

material, if plastic is heated above glass transition temperature and deposited by the extrusion head in the form of layers. This is illustrated in figure 1.

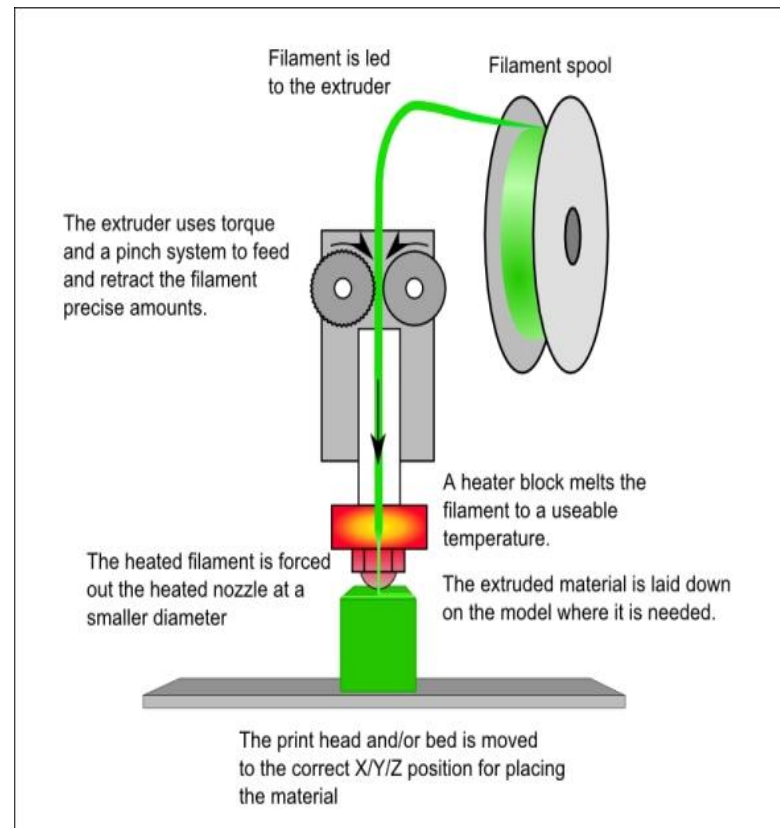

Figure 1: Fused Deposition Modeling principle[4]

There are many commercially available 3D printers using this principle. In this work, Tevo Tarantula i3 Desktop 3D printer (figure 2) is used.

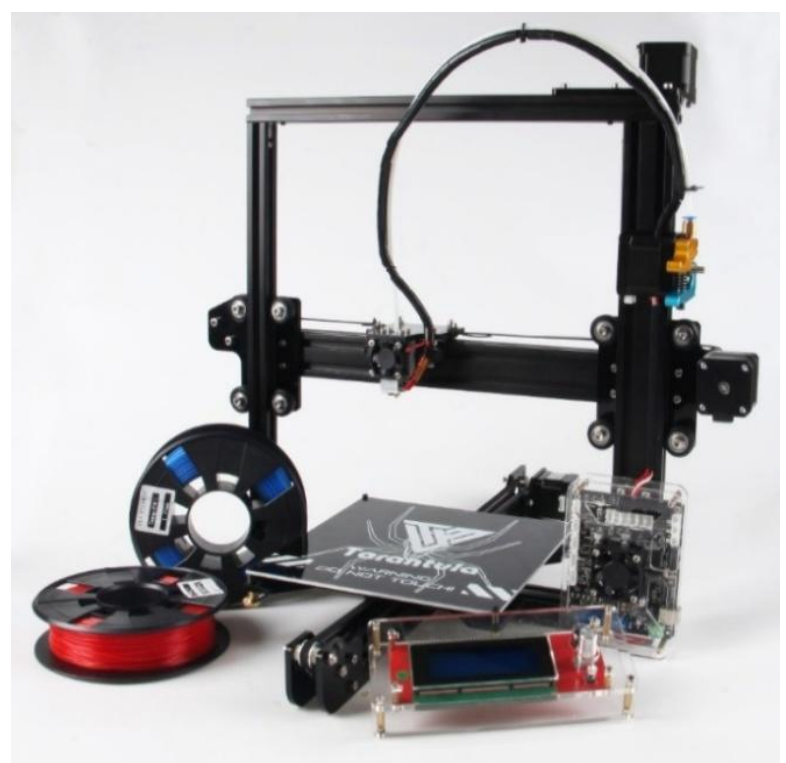

Figure 2: Tevo Tarantula i3 desktop printer[5]
These software use various slicing engines (algorithms) like Cura, Slicer3D etc. In the current work, 'RepetierHost' 3D printing software with 'Cura' as slicing algorithm is used. Figure 3 shows an example of sliced component giving the toolpath of the extruder.

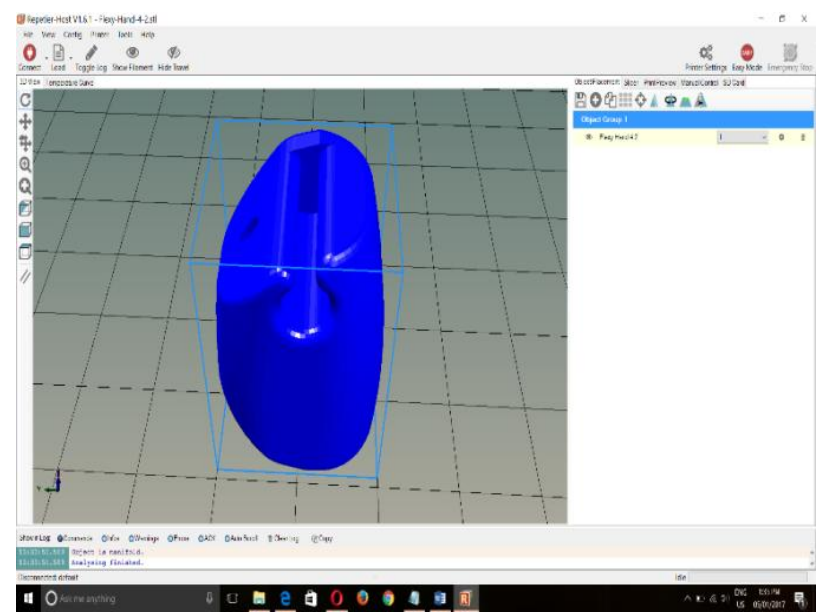

Figure 3: Sliced components in Repetier-Host software [3]

It may be noted here that the $3 \mathrm{D}$ printed components are basically are shells with infills. Various infill patterns like grids, lines, Honeycombs, 3D Honeycombs exist and optionsfor these are available based on the slicing engines/algorithms chosen.

\section{PROSTHETIC HAND}

As mentioned earlier, the aim of the current work is to design and fabricate support for a disabled hand (due to neurological disorder or accidents). 3D models of the components are initially generated using 3D scanners editors and Unigraphics. It looks exactly like Flexy hand.

Figure 4 shows the model of the prosthetic hand. Each component is exported into 'stl' format. The stl files are then imported into Repetier-Host software where slicing of the components is done to generate 3D printing tool path generation and thus the G-Code for the same. Cura Slicing engine is used during slicing process.

Wall thickness of the components is maintained as $0.8 \mathrm{~mm}$ and infill pattern is chosen as 'Grid'. 20\% infill is maintained during 3D printing. Water and adhesive mixture is selected for Adhesion Type and the 3D printing is done in such a way that no supports are required. Figure 5 shows the image during printing of finger. 


\section{IAR JSET}

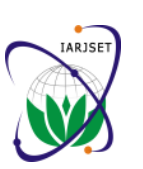

International Advanced Research Journal in Science, Engineering and Technology

National Conference on Design, Manufacturing, Energy \& Thermal Engineering (NCDMETE-2017) AGTI's Dr. Daulatrao Aher College Engineering, Vidyanagar Extension, Karad

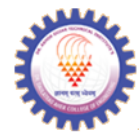

Vol. 4, Special Issue 1, January 2017

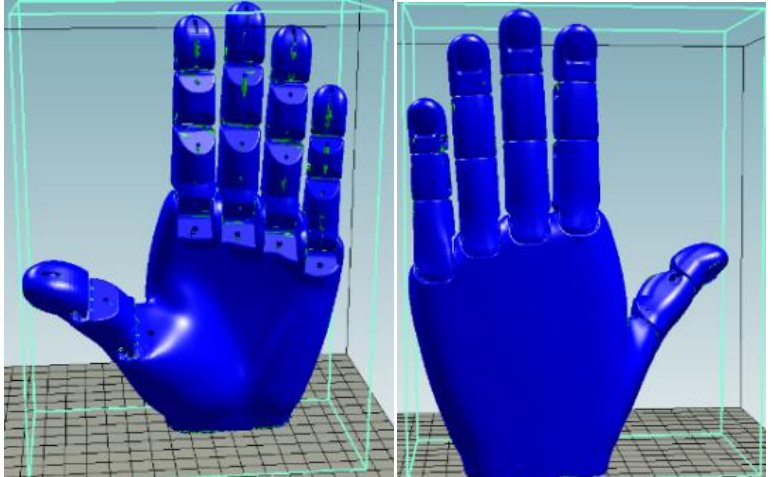

Figure 4: Proshetic hand model design

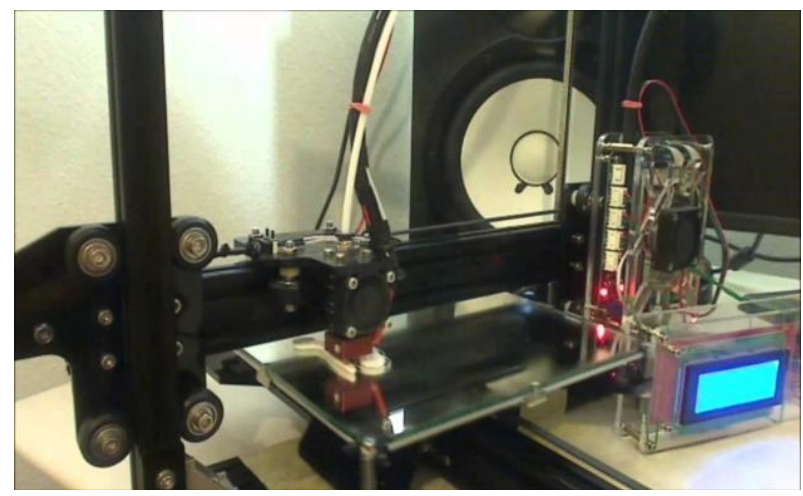

Figure 5: Tevo tarantula printing.

The overall end product is shown in figure 6 below.

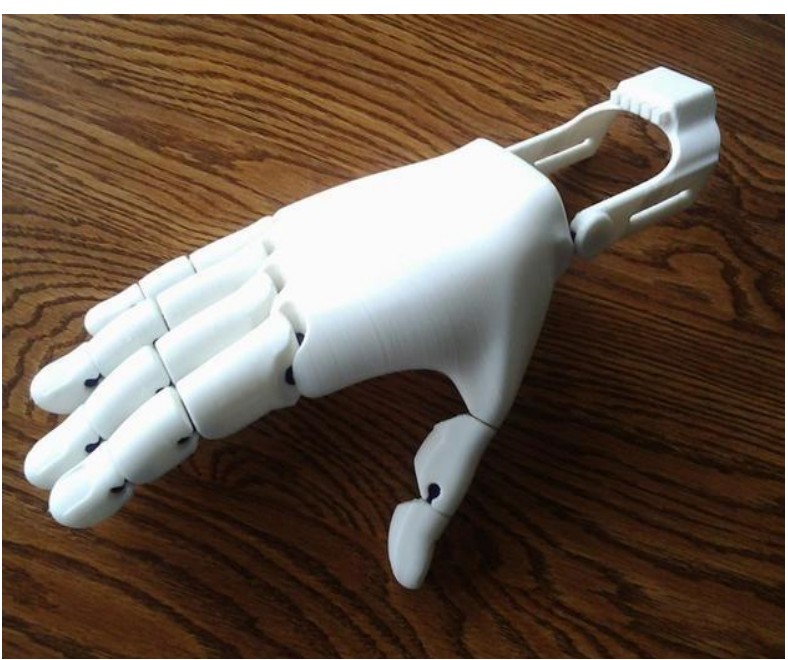

Figure 6: End product

\section{WORKING OF THE PROSTHETIC ARM.}

The working of the prosthetic arm is very simple the prosthic arm is woven with an elastic band which is then attached to the end section and tensioner attached to the hand and it is further connected to an working circuit with the low RPM DC motor with initiates on muscle movement of the arm.

The figure 7 show the mechanism of working.

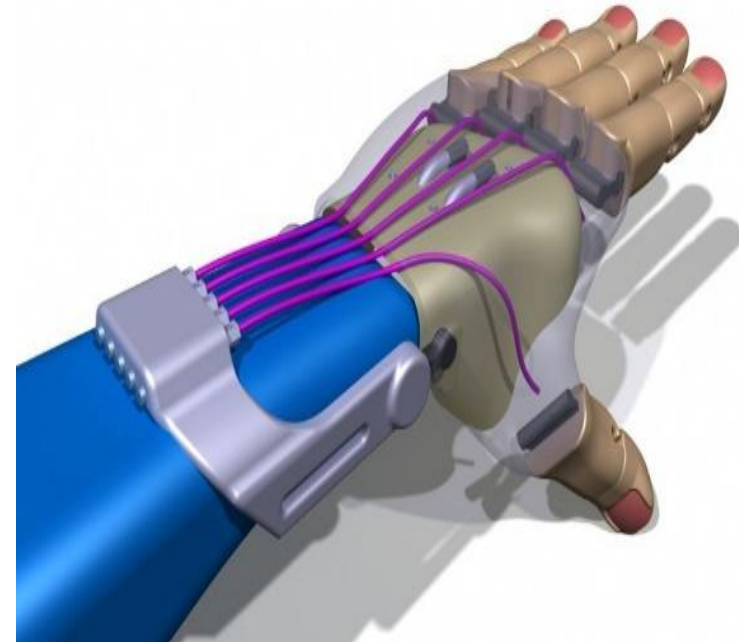

Figure 7: Mechanism of working.

The end product were primary tested for basic movements shown in figure 8 below

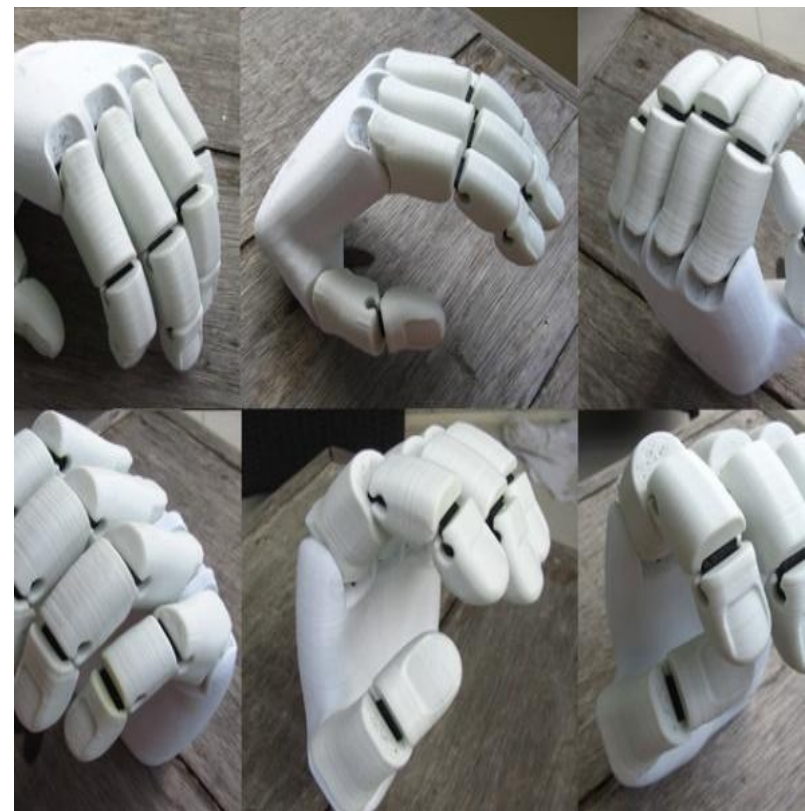

Figure 8: shows various actions performed using the prosthetic arm

Various sensors like flex sensor, pressure sensor were used to sense muscle movement and an arduino circuit for mechanism control was also developed Approximately about $4-5 \mathrm{~kg}$ of load can be lifted using this hand which is quite a good results.

\section{APPLICATION OVER A ROBOT}

The further part of this work included the prosthetic arm to be mounted over the robot.

Figure 9 show an tentative approach over the mounting of arm over the robot. 


\section{IAR JSET}

Vol. 4, Special Issue 1, January 2017

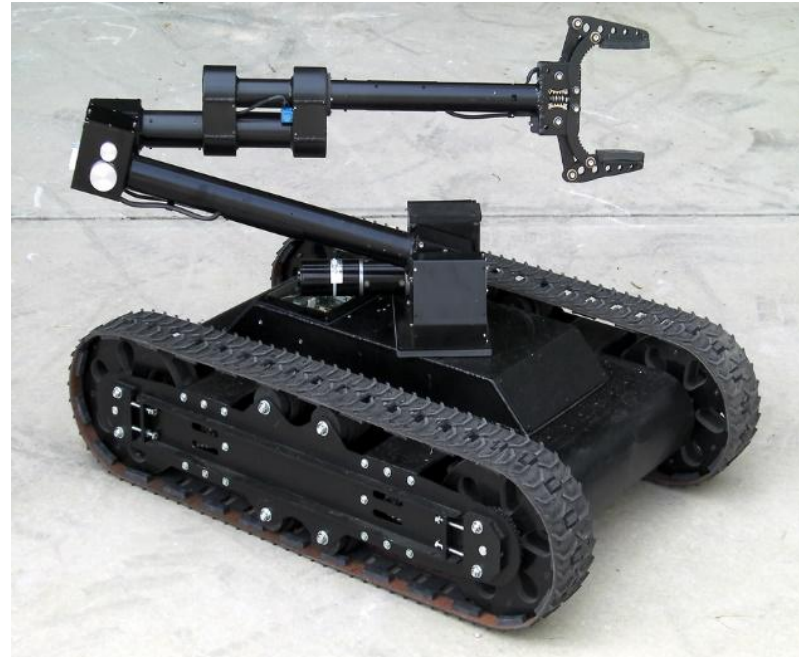

Figure 9: Mounting the Arm

As per the figure various motors and movements controlers are used for the movement of the arm and robot.high degree of freedoms are given to cope with various tenious jobs to be done.Figure 10 shows movements of the robotic arm

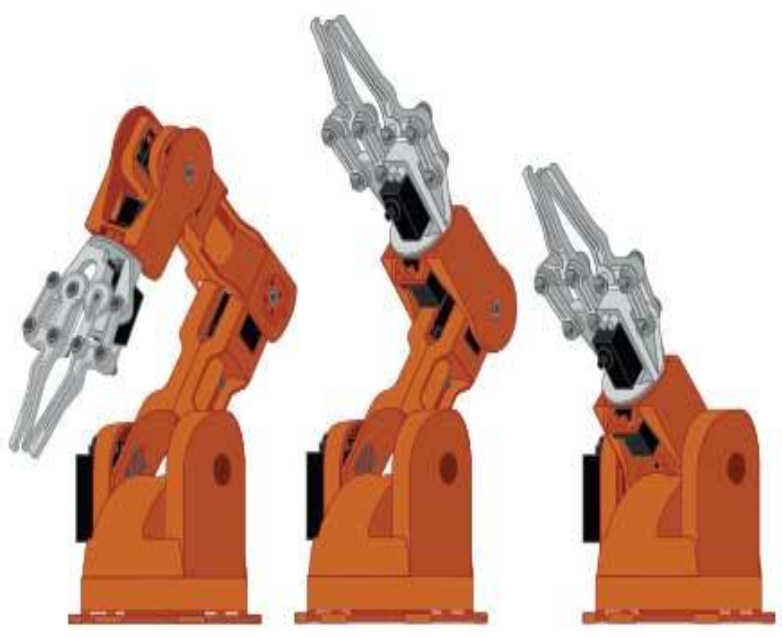

Figure 10: Movements of robotic arm.

The robotic movements are fully programmable hence can be made to work as per our needs. Vast number of applications can be generated using such a robot.

The industrial applications can be stated as follows:

\section{1) WELDING ROBOT APPLICATION}

- Arc Welding

- Electron Beam

- Flux Cored Welding

- Laser Welding

- Mig Welding

- Plasma Cutting

- Plasma Welding

- Resistance Welding
- Spot Welding

- Tig Welding

- TIP/TIG Welding

- Welding Automation

2) MATERIAL HANDLING ROBOT APPLICATIONS

- Collaborative

- Dispensing

- Injection Molding

- Machine Loading

- Machine Tending

- Material Handling

- Order Picking

- Packaging

- Palletizing

- Part Transfer

- Pick and Place

- Press Tending

- Vision

3) OTHER ROBOT APPLICATIONS

- 3D Laser Vision

- Appliance Automation

- Assembly

- Bonding / Sealing

- Cleanroom

- Coating

- Cutting

- Deburring

- Drilling

- Fiberglass Cutting

- Foundry

- Grinding

- Material Removal

- Meat Processing Automation

- Milling

- Painting Automation

- Polishing

- Refueling

- Routing

- Spindling

- Thermal Spray

- Waterjet

\section{CONCLUSION}

Prosthetics, in the current medical industry, involves making of artificial limbs for those who have lost their natural ones. Very little work has been done in the field of making prosthetic supports for hands for those whose disability is primarily due to neurological disorder. 3D printing technology offers in designing custom solutions to these kinds of problems. In the current work, a novel design of prosthetic hand is proposed for those whose 


\section{IAR JSET}

disability is primarily due to neurological disorders or accidents. The designed components are 3D printed and assembled. Also the solution developed is not only applicable to prosthetics the arm is fully compatible for Industrail use also. Also the basic cost of prosthetic arm is about 50000-5000000 INR but this prosthetic arm is produced about 2000-4000 INR only. Hence could be a good option for budget oriented user

\section{REFERENCES}

[1] Dr. Shyam Thangaraju, Vishal Chaudhary (2014), "Application of 3D Printing in Health Care", Engineering And R\&D Services, HCL

[2] C. Lee Ventola (2014), "Medical Applications for 3D Printing: Current and Projected Uses", PT Community Journal, 39(10), pp. 704-711

[3] 3D Printing, Resource URL: https://en.wikipedia.org/wiki/3D_printing\#General_prin ciples

[4] Fused deposition modeling, Resource URL: https://en.wikipedia.org/wiki/Fused_deposition_modeli ng.

[5] www.kmstsa.org, "3D Printed Techologies", http://www.kmstsa.org/technologies.html 